\title{
0641 INCREASING VISIBILITY OF VIOLENCE PREVENTION: LINKING STRATEGIES TO PREVENT VIOLENCE AND PROMOTE HEALTHY EATING AND ACTIVE LIVING
}

L Cohen*, V Lee, R A Davis Correspondence: Prevention Institute, 223 Oak Street, Oakland, CA 94607, USA

\subsection{6/ip.2010.029215.641}

Healthy eating and activity practitioners are increasingly noting that violence is undermining the effectiveness of chronic disease prevention strategies. Prevention Institute has conducted community and academic research to gain an understanding about the relationship between violence and healthy eating and activity and solutions for addressing these complex issues. Additionally, the Institute is coordinating six United States pilot sites in an effort to reduce violence and promote healthy eating and activity in local communities. This work is supported by Kaiser Permanente and the Healthy Eating Active Living Convergence Partnership. Violence negatively affects determinants of health such as where people live and shop, parents not letting their children play outside, and avoiding walking to school or work, which can exacerbate existing illnesses and increase the risk for disease onset. Violence alters the community environment making it less supportive of healthy eating and activity by reducing social cohesion and disincentivising community investments such as healthy food retail. Further, vulnerable populations are disproportionately impacted by high rates of community violence, and this disparity contributes heavily to overall health inequities. Given rising concerns about food- and activity-related chronic disease, this growing recognition of the impact of violence on outcomes presents an opportunity to bring attention to efforts in preventing violence. This presentation provides the unique perspective of a community voice that affirms the growing research base, delineates emerging strategies and the roles for violence prevention and healthy eating and activity practitioners, and highlights opportunities for strategic partnerships between them to create safe and equitable communities. 